\title{
Development of a Composite Electrospun Polyethylene Terephthalate-Polyglycolic Acid Material: Potential Use as a Drug-Eluting Vascular Graft
}

\author{
Christoph S. Nabzdyk, ${ }^{1}$ Maggie Chun, ${ }^{1}$ Saif G. Pathan, ${ }^{2}$ David W. Nelson, ${ }^{2}$ Jin-Oh You, ${ }^{3,4}$ \\ Matthew D. Phaneuf, ${ }^{2}$ Frank W. LoGerfo, ${ }^{1}$ and Leena Pradhan-Nabzdyk ${ }^{1}$ \\ ${ }^{1}$ Division of Vascular and Endovascular Surgery, Beth Israel Deaconess Medical Center, Harvard Medical School, Boston, \\ MA 02215, USA \\ ${ }^{2}$ BioSurfaces, Inc., Ashland, MA 01721, USA \\ ${ }^{3}$ Harvard School of Engineering and Applied Sciences, Cambridge, MA 02138, USA \\ ${ }^{4}$ Department of Engineering Chemistry, Chungbuk National University, Cheongju, Chungbuk 28644, Republic of Korea
}

Correspondence should be addressed to Leena Pradhan-Nabzdyk; lpradhan@bidmc.harvard.edu

Received 15 April 2015; Revised 24 July 2015; Accepted 3 August 2015

Academic Editor: Mircea Chipara

Copyright (C) 2015 Christoph S. Nabzdyk et al. This is an open access article distributed under the Creative Commons Attribution License, which permits unrestricted use, distribution, and reproduction in any medium, provided the original work is properly cited.

Intimal hyperplasia (IH), an excessive wound healing response of an injured vessel wall after bypass grafting, typically leads to prosthetic bypass graft failure. In an approach to ameliorate IH, nondegradable poly(ethylene terephthalate) or PET, which has been used in prosthetic vascular grafts for over 60 years, and biodegradable poly(glycolic acid) or PGA were electrospun using different techniques to generate a material that may serve as permanent scaffold and as a drug/biologic delivery device. PET and PGA polymers were electrospun from either a single-blended solution (ePET/ePGA-s) or two separate polymer solutions (ePET/ePGAd). ePET/ePGA-d material revealed two distinct fibers and was significantly stronger than the single fiber ePET/ePGA-s material. After 21 days of incubation in PBS, ePET-PGA-s showed fiber strand breaks likely due to the degradation of the PGA within the ePET-ePGA-s fiber, while the ePET/ePGA-d material showed intact ePET fibers even after ePGA fiber degradation. The ePET/ePGA- material was able to release red fluorescent dye for at least 14 days. Attachment of human aortic smooth muscle cells (AoSMCs) was similar to both materials. ePET/ePGA-d materials maybe a step towards bypass graft materials that can be custom-designed to promote cellular attachment while serving as a drug delivery platform for IH prevention.

\section{Introduction}

Prosthetic bypass graft failure is most frequently a result of intimal hyperplasia (IH) at the distal anastomosis [1]. $\mathrm{IH}$ is the result of an excessive wound healing response of an injured vessel wall after bypass grafting or angioplasty. Hallmarks of IH are endothelial activation followed by the ad luminal migration of synthetic smooth muscle cells that secrete significant amounts of extracellular matrix. This eventually leads to a hemodynamically relevant luminal narrowing of the conduit. Poly(ethylene terephthalate) or PET has been used for arterial bypass grafts for over 60 years in woven or knitted constructs, both of which require sealants to prevent blood seepage through the graft wall. None of these structural variants have provided a scaffold that mimicked extracellular matrix (ECM), a major component of a blood native vessel onto which cells can grow.

Electrospinning is a versatile technology that can yield nanofiber materials. Interestingly, the materials' ultrastructure resembles that of ECM [2]. Electrospinning can be used for controlled fiber modification and nanocomposite substrate integration $[3,4]$. The materials' ultrastructure characteristically consists of crisscrossing nano- to micrometer thick fibrils, resembling extracellular matrix. This ultrastructure not only results in a large surface area, which could be utilized for drug delivery, but also allows cellular trafficking. 
Aside from optimizing prosthetic bypass grafts, researchers have attempted to prevent $\mathrm{IH}$ formation via drug release from the prosthetic graft surface [5].

Microarray analysis of developing canine IH lesions identified various target genes that were dysregulated for up to four weeks after bypass graft implantation [6]. RNAi interference may become a promising technology that may help modulate expression of genes contributing to $\mathrm{IH}$ and other cardiovascular diseases [7-9]. As cellular signaling networks are highly redundant, it may be necessary to modulate/silence multiple genes in either a synchronous or metachronous fashion. In a previous study from our group, short-term RNAi release from dip-coated electrospun PET grafts was shown to significantly silence target gene-expression of infiltrating human aortic smooth muscle cells [2]. siRNA dip-coating of ePET did not interfere with cell adhesion, infiltration, or cell viability [2].

The next logical step was to introduce a degradable component into the ePET material using electrospinning technology that could act as reservoir for siRNA or other therapeutic compounds with the goal to prolong siRNA release from within the material. Poly(glycolic acid) or PGA, a biocompatible ester that degrades predictably by ester hydrolysis, appears to be a good candidate biomaterial to provide localized drug delivery. The final construct must meet the following requirements: (1) degradable component has to completely degrade over a predictable period of time, (2) composite material has to retain its backbone structure of robust ePET, (3) introduction of a degradable component should not interfere with cell attachment, and (4) therapeutic compound should be preferentially stored in the degradable component and not in the nondegradable ePET.

The goal of this study is to design and evaluate a composite electrospun material that consists of a nondegradable ePET backbone and interspersed degradable polyglycolic acid (PGA) fibers for biodegradation, simulated drug release, and cell attachment.

\section{Methods}

2.1. Electrospinning of ePET/ePGA Materials. Electrospinning of ePET, ePGA, single solution ePET/ePGA (ePET/ ePGA-s), and dual solution ePET/ePGA (ePET/ePGA-d) was done using a computer-automated electrospinning apparatus. PET (10\% w:v) and/or PGA (10\% w:v) polymer chips were dissolved in hexafluoroisopropanol (HFIP) and mixed for a minimum of 48 hours on an inversion mixer at $45 \mathrm{rpm}$. The PET, PGA, PET/PGA-s (blended), and PET/PGA-d (electrospun at the same time from different solutions) were each electrospun using a steady rate of $3 \mathrm{~mL}$ solution/hour at $+20 \mathrm{kV}$ for 60 minutes. The resulting electrospun materials were collected onto a Teflon-coated mandrel $(30 \mathrm{~cm}$ diameter). In another set of experiments, DyLight 549 was added to the PGA and PET solutions, respectively, prior to electrospinning. The electrospun materials were dried for 48 hours at $40^{\circ} \mathrm{C}$ at $99.9 \%$ vacuum. All materials were sterilized via ethylene oxide at $25^{\circ} \mathrm{C}$ for 12 hours under humidified conditions in an Anprolene Sterilizer. Expanded polytetrafluoroethylene (PTFE), one of the more commonly used materials for prosthetic vascular grafts, served as a clinical reference material for the surface morphology and cell culture studies.

2.2. Evaluation of Tensile Strength of ePET, ePGA, ePET/ PGA-s, and ePET-PGA-d Materials. Sheets of ePET, ePGA, ePET/ePGA-s, and ePET/ePGA-d were cut into rectangular strips $(20 \mathrm{~mm} \times 5 \mathrm{~mm} ; n=5$ segments/test group/test condition), with thickness measured using an Ames Thickness Gauge. Strength testing was carried out using a Q-Test Tensile Strength apparatus (MTS Systems, Cary, NC) with a $25 \mathrm{lb}$ load cell and clamps set at a gap distance of $5 \mathrm{~mm}$. The clamp traverse speed was set at a $200 \mathrm{~mm} / \mathrm{min}$ strain rate until break.

2.3. Degradation and DyLight 549 Release Studies. $5 \times 5 \mathrm{~mm}^{2}$ segments of ePET, ePET/ePGA-s, and ePET/ePGA-d materials were placed in sterile phosphate buffered saline (PBS) and incubated at $37^{\circ} \mathrm{C}$ on an orbital shaker for up to 21 days. Samples were rinsed, air-dried, and evaluated using scanning electron microscopy (SEM) or confocal imaging, respectively, using standard techniques.

2.4. Quantification of AoSMC Attachment to PTFE Electrospun Material. Primary human aortic smooth muscle cells (AoSMCs) (Lonza, Walkersville, MD) (passages 4-8) were cultured as described previously [10]. $5 \times 5 \mathrm{~mm}^{2}$ segments of each material were cut and placed into 96-well tissue culture plates. AoSMCs (40,000 cells/well) were seeded on top of PTFE, ePET, ePET/ePGA-s, and ePET/ePGA-d materials and allowed to attach. After 3 hours, segments were removed, rinsed, and placed in a new well. alamarBlue ${ }^{\circledR}$ assay was then used to assess cell attachment $[2,10]$. AoSMC attachment to the tissue culture well alone served as the positive attachment control and Alamar blue within an empty well served as a negative control.

2.5. Statistical Analysis. One-way analysis of variance (ANOVA) with Bonferroni post hoc correction was applied. A $p$ value $<0.05$ was considered significant.

\section{Results}

3.1. Fiber Morphology. While the PTFE graft material consisted of predominantly parallel-aligned fibers with little interfiber space arranged between thick nodes (Figure 1(a)), the ePET material consisted of a fiber mesh with variable interfiber spaces with relatively reduced fiber diameter distribution (Figure 1(b)). ePGA, ePET/ePGA-s, and ePET/PGA-d fibers also showed distinct fiber strand architecture (Figures 1(c)-1(f); left panel).

3.2. Polymer Degradation Using SEM. Degradation studies of the ePGA material alone over a 21-day period revealed initial fiber swelling and gradual degradation (Figures 1(c) and $1(\mathrm{~d})$; middle and right panel). Degradation of ePETPGA-s material resulted in strand breaks between 14 and 21 days (Figure 1(e); middle and right panel) whereas the ePET/ePGA-d material showed that only ePGA fibers 


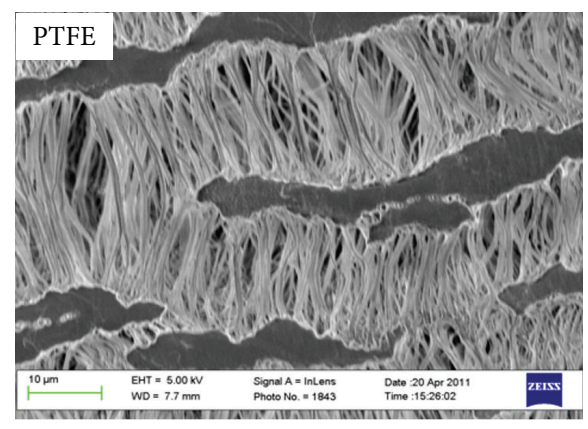

(a)

Day 1
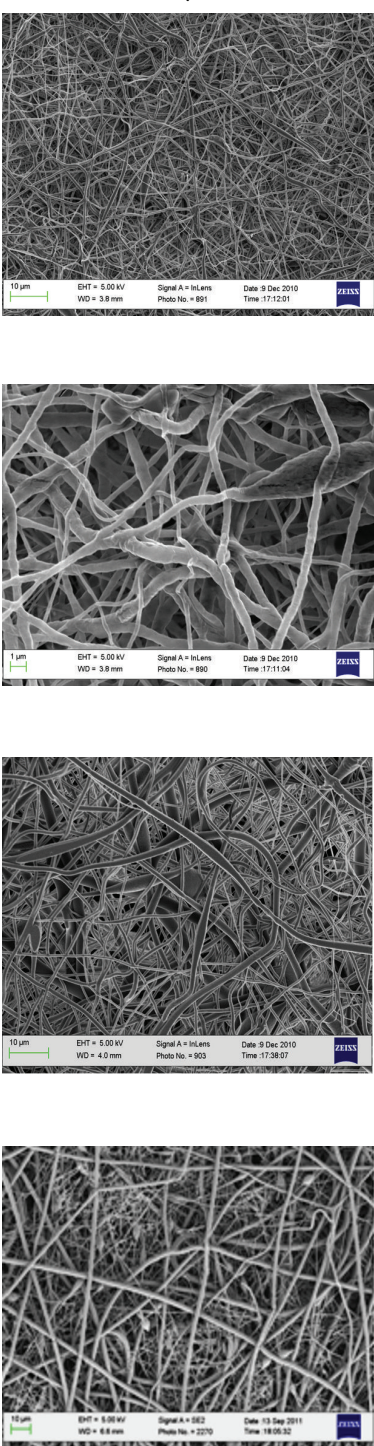

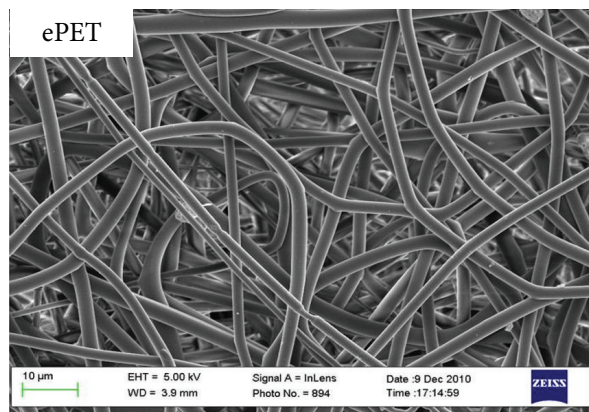

(b)

Day 21
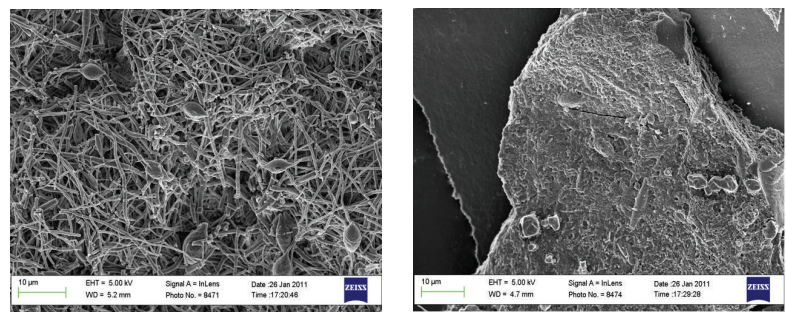

ePGA

(low mag.)

(c)
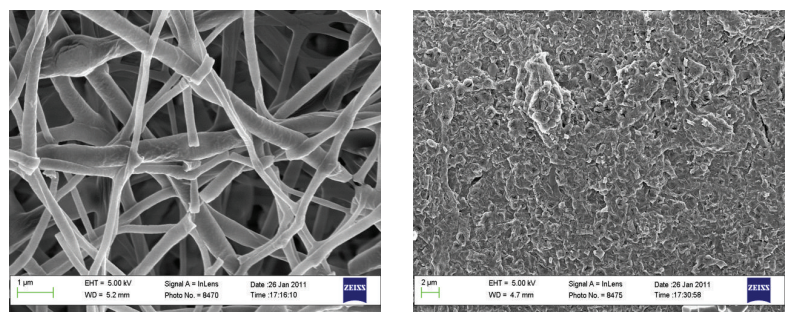

ePGA

(high mag.)

(d)
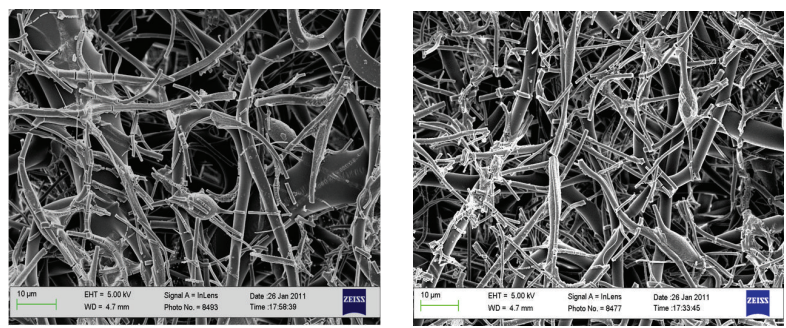

ePET/ePGA-s

(e)
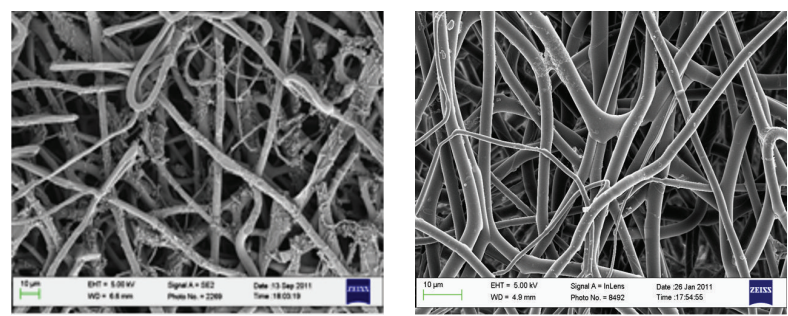

ePET/ePGA-d

(f)

FIGURE 1: Evaluation of surface morphology of control and electrospun materials via SEM: representative SEM pictures of PTFE and ePET (a and b). SEM analysis of ePGA fiber degradation as a single or a composite material with PET. ePGA fibers gradually degrade over 14-21 days in PBS ( $c$ and d). In contrast, after ePGA degradation the ePET/ePGA-s (e) or ePET/ePGA-d materials (f) still have a fibrous composition due to the nondegrading ePET fibers. Note the strand breaks in the ePET/ePGA-s group (presumably due to PGA degradation) while no strand breaks occurred in the ePET/ePGA-d group. 


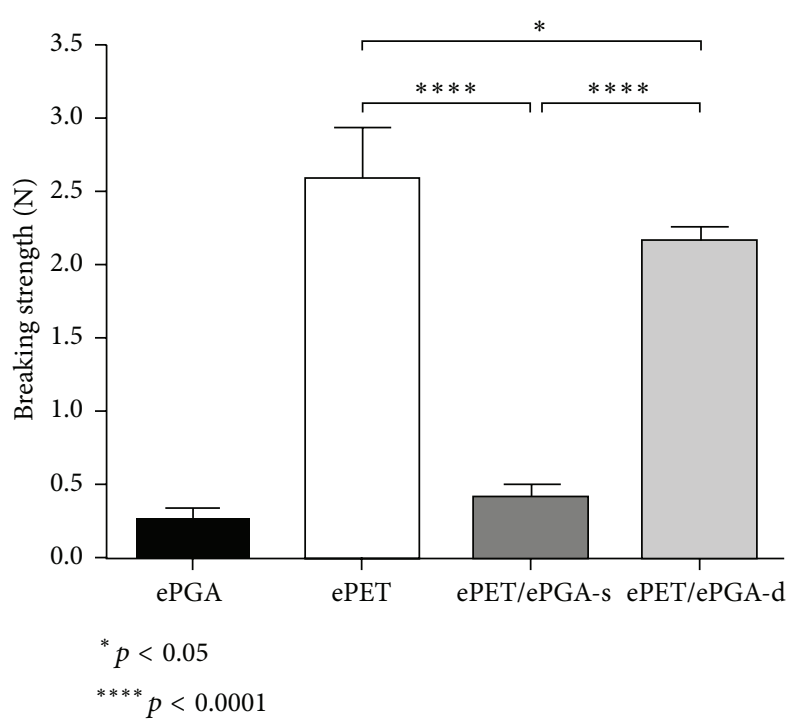

FIGURE 2: Evaluation of tensile strength of the electrospun materials: electrospun materials were evaluated for tensile strength. ePET is the strongest material, while ePGA is the weakest. ePET/ePGA-d had significantly higher strength as compared to ePET/ePGA-s.

degrade over time, leaving an intact ePET fiber network behind (Figure 1(f); middle and right panel) that closely resembles the network of single strand ePET fibers seen in Figure 1(b). ePET/ePGA-d thus has the potential of retaining all of its strength meanwhile allowing more porous architecture for cells to infiltrate and heal the graft as the electrospun PGA component degrades and releases the active siRNA.

3.3. Tensile Strength. ePET materials had significantly more strength $(2.57 \pm 0.34 \mathrm{~N})$ as compared to ePET/PGA-d (2.16 \pm $0.10 \mathrm{~N})$ and ePET/PGA-s $(0.42 \pm 0.07 \mathrm{~N})$ materials, respectively ( $p \leq 0.0001$ ) (Figure 2). Maintaining the ePGA as a separate fiber allowed the ePET/PGA-d material to retain a majority of its strength as compared to ePET/PGA-s, which was significantly weaker. ePGA material alone $(0.25 \pm 0.08 \mathrm{~N})$ was the weakest of all of the materials evaluated. We also observed that, compared to ePET/PGA-d, ePET had a slightly higher (19\%) strength.

\subsection{Identification of ePGA Fiber Location via Using Fluo-} rescent Labeling. A clear separation between fluorescently labeled ePGA and nonlabeled ePET strands can be seen in the ePET/ePGA-d materials indicating no cross-reaction between the two individual polymers (Figure 3(a)). The electrospinning procedure allowed for adjustable fiber ratio within the length of the ePET/ePGA-d polymer (Figure 3(b)).

3.5. DyLight 549 Release from ePGA. Confocal imaging showed that DyLight 549 was released from ePGA of the ePET/PGA-d polymer over 14 days (Figure 4 ). This DyLight 549 release profile correlated well with the observed degradation rate of the ePGA fibers.

3.6. Cell Attachment. AoSMC attachment to both ePET and ePET/ePGA-d polymers was significantly higher as compared to PTFE (Figure 5). AoSMC attachment to all of the electrospun materials was higher than that of culture plate. The introduction of ePGA fibers in both of the composite polymers did not appear to alter the attachment to the ePET backbone fiber mesh.

\section{Discussion}

Electrospinning can be used to generate various materials from different polymers $[3,4]$. In this study, coelectrospinning resulted in a composite material of a nondegradable and a degradable polymer without polymer cross-reaction. Coelectrospinning of the PET with PGA results in the least reduction of tensile strength as compared to a single solution electrospin. This strength data validates that blending the solutions results in comingling of fibers that may ultimately reduce overall device strength.

More importantly, a compound can be incorporated and released upon ePGA degradation as indicated by DyLight release. The molecular weight of the DyLight molecule is similar to that of bioactive agents such as antiproliferative drugs and siRNA. Even after PGA degradation, there were no strand breaks observed in ePET/ePGA-d materials after 21 days in solution, which is reassuring in regard to the tensile strength of the polymer. Given that ePET/ePGA-d polymer contains a nondegradable and load-bearing component in the form of ePET, mechanical failure of the proposed graft is not a concern, especially when compared to fully degradable bypass grafts $[3,11,12]$.

AoSMC attachment was not affected when ePGA was introduced into the composite polymer. This is an important finding, as an ideal graft design should promote initial cell attachment and growth. Further, the electrospinning protocols allowed for fiber density variation within the coelectrospun polymer, which could be used to generate therapeutic compound gradients within the graft. 

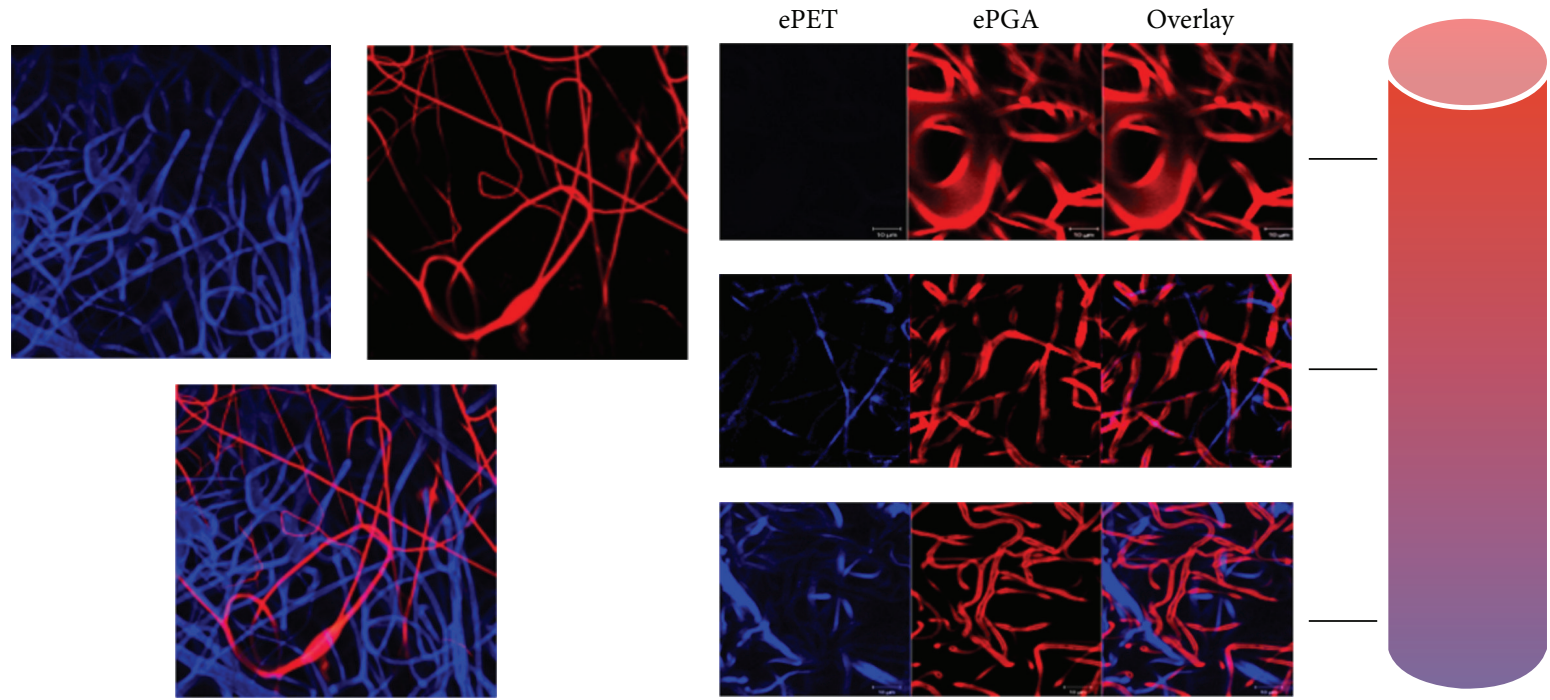

- ePET fibers in ePET/ePGA-d
ePGA fibers in ePET/ePGA-d

(a)
- ePET fibers in ePET/ePGA-d
ePGA fibers in ePET/ePGA-d

(b)

Figure 3: Spatial fiber distribution in coelectrospun ePET/ePGA-d polymer using DyLight labeling and adjustable fiber ratio within the graft material: confocal microscopy images of fluorescently labeled coelectrospun ePET/ePGA-d polymer reveal sharp fiber and compound separation (a). Blue fluorescent filter highlights ePET fibers within the coelectrospun ePET/ePGA-d polymer. Red fluorescent filter highlights DyLight 549 containing ePGA fibers within the ePET/ePGA-d material. Representative images of three separate areas within the length of coelectrospun material with varying fiber ratio (b). (Top row) Only DyLight 549 containing ePGA fibers are present. (Middle row) A few blue ePET fibers are intertwined with the ePGA fibers. (Bottom row) A roughly equal fiber frequency of ePET and ePGA is present.

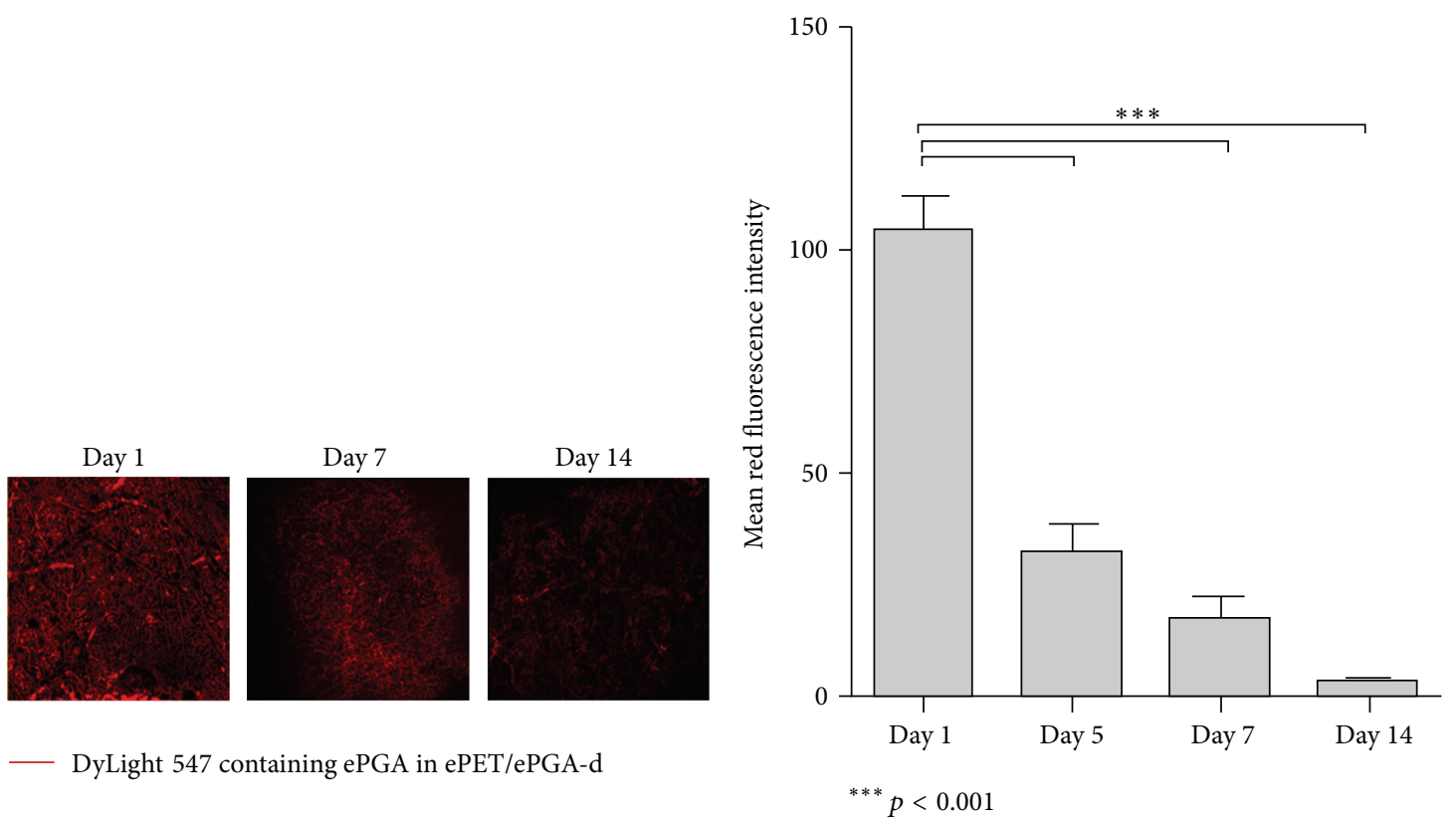

(a)

(b)

FIGURE 4: DyLight release from ePGA fibers of ePET/ePGA-d polymer: confocal imaging of ePET/ePGA-d polymer with ePGA fibers containing DyLight 549 (a). Degradation study shows steady release of DyLight 549 from the ePGA fibers of ePET/ePGA polymer for at least 14 days (b). 


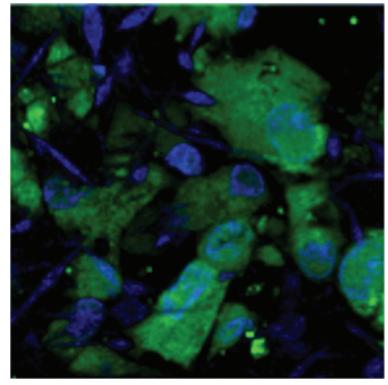

ePET/ePGA-s

_- Hoechst nuclei stain

— Cell tracker green AoSMC

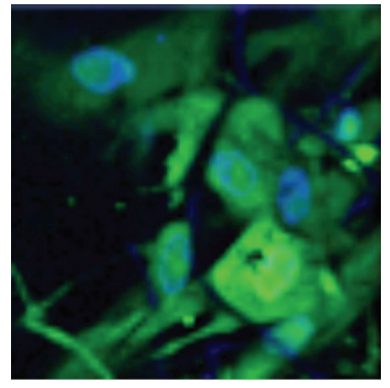

ePET/ePGA-d

(a)

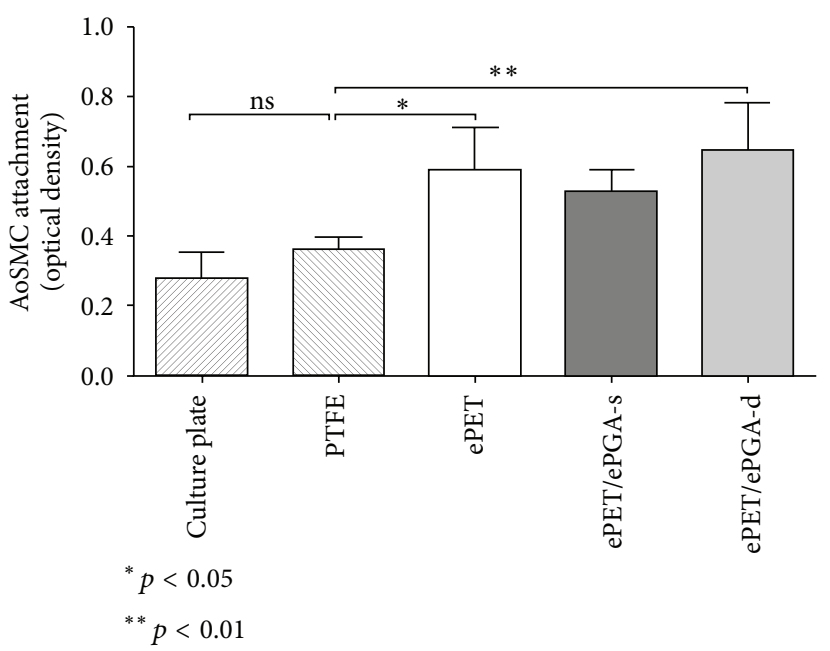

(b)

Figure 5: AoSMC attachment to electrospun materials: confocal imaging (a) and Alamar blue assay evaluating AoSMC attachment to composite electrospun polymers (b). AoSMC attachment was greatest in the electrospun materials as compared to the attachment on the PTFE and tissue culture plate, respectively. There was no significant difference in AoSMC binding between the electrospun materials.

This data provides first evidence that coelectrospinning of ePET/ePGA may be a viable option for sustained local drug delivery from within a prosthetic arterial bypass graft. This technology will be combined in future experiments with our previously published approach for short-term siRNA release from electrospun PET materials [2]. Additional studies will extensively analyze drug/siRNA loading and delivering capacities, biocompatibility, and tensile strength in vitro and in vivo to evaluate the material's properties as drug delivering vascular prostheses. Introduction of other polymers such as poly(lactic-co-glycolic acid) (PLGA) and polycaprolactone (PCL) with slower degradation rates than PGA may aid in providing sequential and even more sustained drug/siRNA release from the material $[3,11,12]$ while possibly adding a stronger polymer to the cospin. These polymer moderations could act synergistically to comprehensively modulate the tissue response to material implantation. This approach may become a platform technology for various biomaterial modifications and applications for implantable surgical devices and possibly arterial bypass grafts.

\section{Conflict of Interests}

The authors declare that there is no conflict of interests regarding the publication of this paper.

\section{Acknowledgments}

This work was supported by NIH 5R01 HL021796-30, NIH 2R01 HL086741-08, T32 HL007734-21, and the William J. von Liebig Foundation funds to Frank W. LoGerfo.

\section{References}

[1] V. S. Sottiurai, J. S. T. Yao, W. R. Flinn, and R. C. Batson, "Intimal hyperplasia and neointima: an ultrastructural analysis of thrombosed grafts in humans," Surgery, vol. 93, no. 6, pp. 809-817, 1983.

[2] C. S. Nabzdyk, M. C. Chun, H. S. Oliver-Allen et al., "Gene silencing in human aortic smooth muscle cells induced by PEI-siRNA complexes released from dip-coated electrospun poly(ethylene terephthalate) grafts," Biomaterials, vol. 35, no. 9, pp. 3071-3079, 2014.

[3] S. Wang, X. M. Mo, B. J. Jiang et al., "Fabrication of smalldiameter vascular scaffolds by heparin-bonded P(LLA-CL) composite nanofibers to improve graft patency," International Journal of Nanomedicine, vol. 8, pp. 2131-2139, 2013.

[4] J. Han, S. Farah, A. J. Domb, and P. I. Lelkes, "Electrospun rapamycin-eluting polyurethane fibers for vascular grafts," Pharmaceutical Research, vol. 30, no. 7, pp. 1735-1748, 2013.

[5] C. Cagiannos, O. R. Abul-Khoudoud, W. DeRijk et al., "Rapamycin-coated expanded polytetrafluoroethylene bypass grafts exhibit decreased anastomotic neointimal hyperplasia in a porcine model," Journal of Vascular Surgery, vol. 42, no. 5, pp. 980-988, 2005.

[6] M. Bhasin, Z. Huang, L. Pradhan-Nabzdyk et al., “Temporal network based analysis of cell specific vein graft transcriptome defines key pathways and hub genes in implantation injury," PLoS ONE, vol. 7, no. 6, Article ID e39123, 2012.

[7] S. M. Elbashir, J. Harborth, W. Lendeckel, A. Yalcin, K. Weber, and T. Tuschl, "Duplexes of 21-nucleotide RNAs mediate RNA interference in cultured mammalian cells," Nature, vol. 411, no. 6836, pp. 494-498, 2001.

[8] C. S. Nabzdyk, H. Lancero, K. P. Nguyen, S. Salek, and M. S. Conte, "RNA interference-mediated survivin gene knockdown induces growth arrest and reduced migration of vascular smooth muscle cells," American Journal of Physiology-Heart and Circulatory Physiology, vol. 301, no. 5, pp. H1841-H1849, 2011.

[9] L. Pradhan-Nabzdyk, C. Huang, F. W. LoGerfo, and C. S. Nabzdyk, "Current siRNA targets in atherosclerosis and aortic aneurysm," Discovery Medicine, vol. 17, no. 95, pp. 233-246, 2014. 
[10] S. Yoshida, C. S. Nabzdyk, L. Pradhan, and F. W. Logerfo, "Thrombospondin-2 gene silencing in human aortic smooth muscle cells improves cell attachment," Journal of the American College of Surgeons, vol. 213, no. 5, pp. 668-676, 2011.

[11] S. Chung, N. P. Ingle, G. A. Montero, S. H. Kim, and M. W. King, "Bioresorbable elastomeric vascular tissue engineering scaffolds via melt spinning and electrospinning," Acta Biomaterialia, vol. 6, no. 6, pp. 1958-1967, 2010.

[12] M. Chen, S. Gao, M. Dong et al., "Chitosan/siRNA nanoparticles encapsulated in PLGA nanofibers for siRNA delivery," ACS Nano, vol. 6, no. 6, pp. 4835-4844, 2012. 

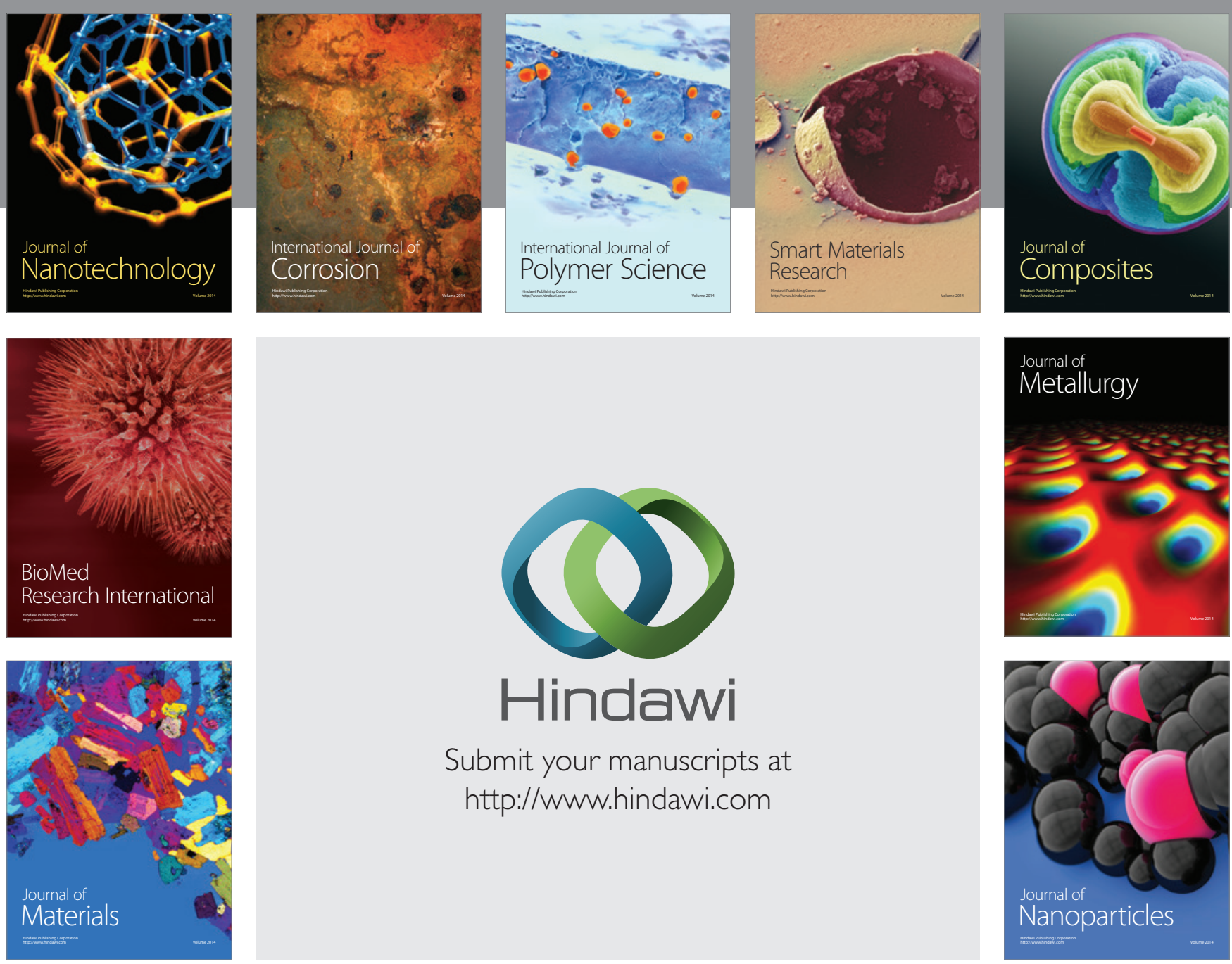

Submit your manuscripts at http://www.hindawi.com
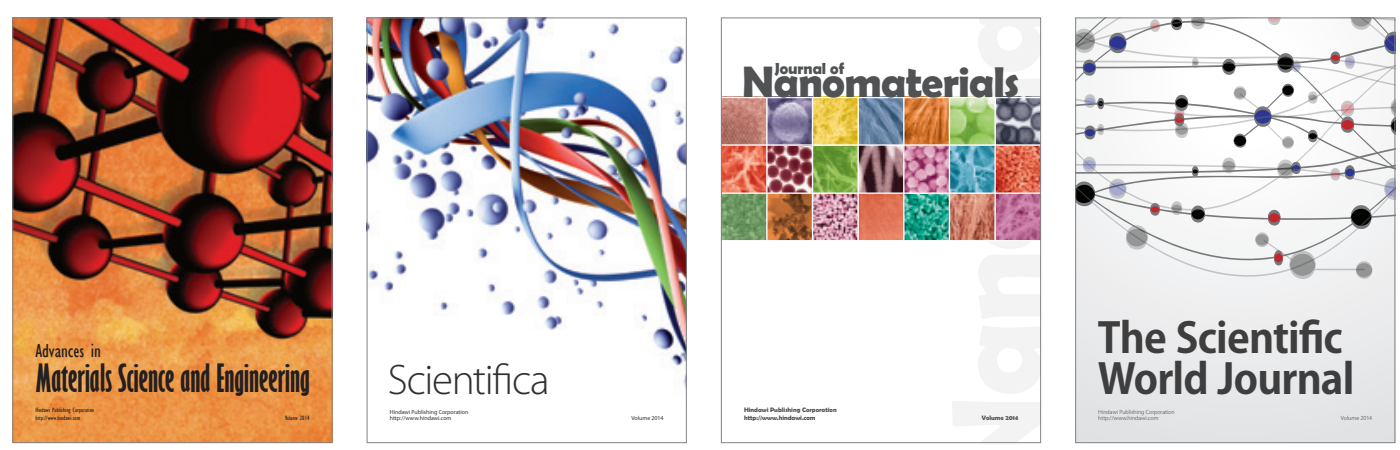

\section{The Scientific World Journal}
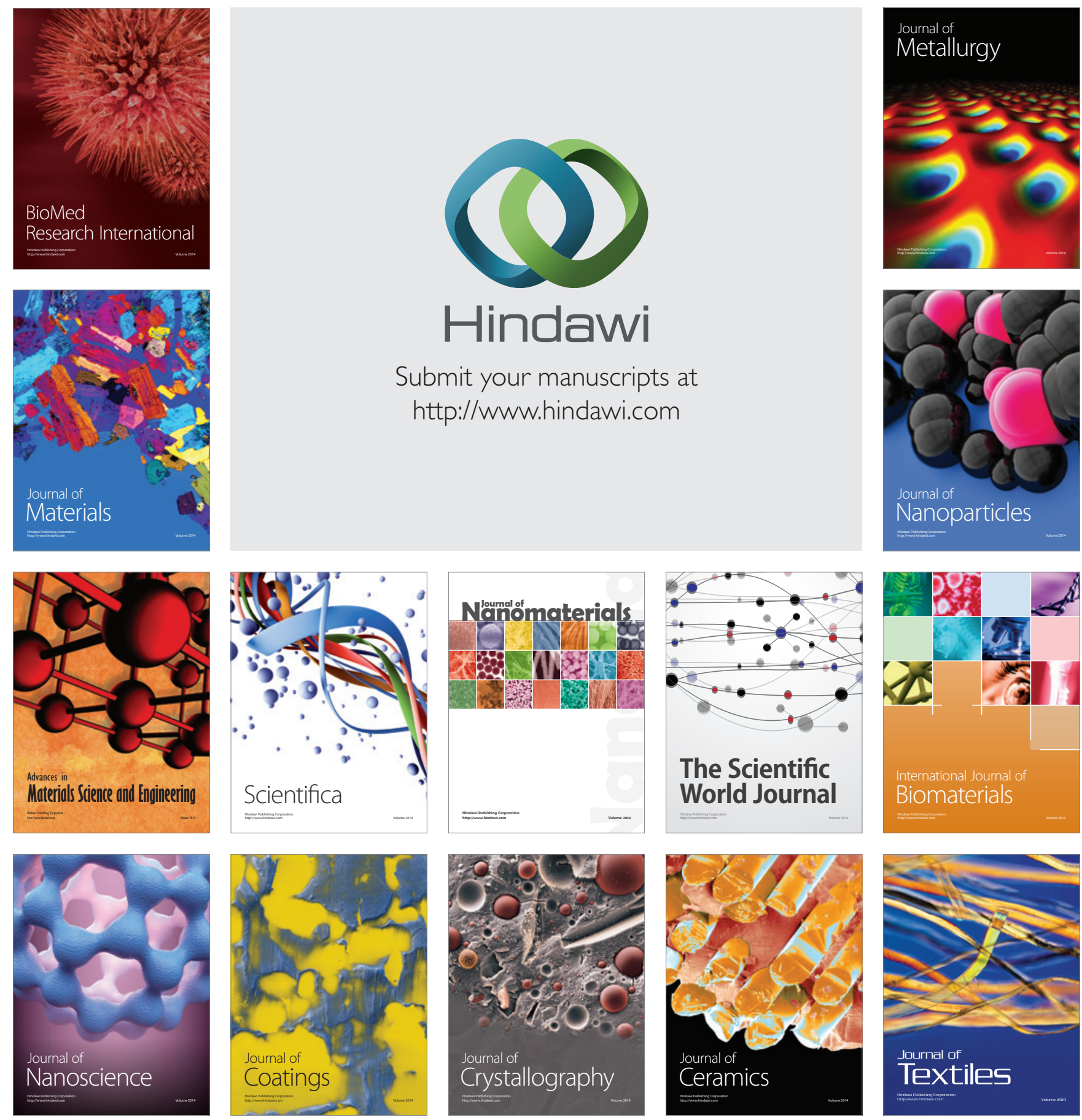